\title{
A New Approach to Fingerprint Detection Using a Combination of Minutiae Points and Invariant Moments Parameters
}

\author{
Sarnali Basak*, Md. Imdadul Islam* and M. R. Amin*
}

\begin{abstract}
Different types of fingerprint detection algorithms that are based on extraction of minutiae points are prevalent in recent literature. In this paper, we propose a new algorithm to locate the virtual core point/centroid of an image. The Euclidean distance between the virtual core point and the minutiae points is taken as a random variable. The mean, variance, skewness, and kurtosis of the random variable are taken as the statistical parameters of the image to observe the similarities or dissimilarities among fingerprints from the same or different persons. Finally, we verified our observations with a moment parameter-based analysis of some previous works.
\end{abstract}

Keywords-Random Variable, Skewness, Kurtosis, Invariant Moment, Termination And Bifurcation Points, Virtual Core Point

\section{INTRODUCTION}

The process of personal fingerprint identification using a ridge skin layout that is used to describe biometric characteristics is known as dactyloscopy. It is one of the most dependable and sensible approaches for recognizing an authorized person from among several masquerades, because the fingerprint features are not changeable and are transient like passwords and cards $[1$, $2]$. The ridge and furrow patterns on the tip/surface of the finger, including bifurcations, termination, and the valley, have been applied in a comprehensive way to determine the uniqueness of the fingerprints of a human being. A Y-shaped junction identifies bifurcations, terminations are the endpoint of the ridges, and the valley is the gap between two ridges.

Usually, the Automatic Fingerprint Identification System (AFIS) performs three basic steps to recognize a fingerprint: preprocessing, feature extraction, and classification, which are explained in $[3,4]$. The overall performance of AFIS mostly relies on extracting features and its efficient matching mechanisms. It still deserves a much more advanced and accessible high volume fingerprint database that takes into account the computational speed and accuracy of matching [5, 6]. To extract and compare of an image for matching, roughly three techniques can be categorized: minutiae-based, correlation-based, and hybrid explained in [7].

In the minutiae-based approach, small and precise portions called "minutiae" represent those

Manuscript received October 27, 2011; accepted June 8, 2012.

Corresponding Author: M. R. Amin

* Department of Computer Science and Engineering, Jahangirnagar University, Savar, Dhaka 1342, Bangladesh (imdad@juniv.edu)

** Department of Electronics and Communications Engineering, East West University, Plot No.A/2, Jahurul Islam City, Aftabnagar, Dhaka 1219, Bangladesh (ramin@ewubd.edu) 
local ridge characteristics, which are singular points (SP) or singularities of the fingerprint. The core and delta are of great consequence sections in SP and for fingerprint classification along with matching $[8,9]$. On the other hand, the correlation-based method broadly deals with texture description [10], ridge shape, and alignment. The principles of both of the methods are used by the hybrid method [11-14].

All of the above methods have more or less several imperfections. Some of these types of imperfections include: the performance of an algorithm and its computational speed degrades when the image quality is not satisfactory, for example the case of a multi-spectral noisy image [5]; a limited efficiency when a different scale position and rotation angle are used for the same input images [15]; or suffering from time-consuming alignment (correlation-based).

A. K. Jain et al. [8] proposed a correlation-based method where the changes in position of the input image are skipped over with respect to a reference point. In [5], J. C. Yang et al. extracted invariant moment properties on a certain Region of Interest (ROI) and an eigen value weighted cosine distance was used to detect similarities or dissimilarities. J. Garcia et al. [16] applied a filtering method and a moment feature to calculate the parameter for matching. M. S. Khalil et. al [17] analyzed by detecting the singular points of an image based on the statistical analysis of the co-occurrence matrix. Zhang et al. [18] proposed a multi-resolution direction field concept to detect the core point for matching. Another core extraction-based method was proposed by A. Mishra [19]. The above technique uses the orientation mask with the neighborhood averaging method to detect the core point. Both the geometric moment and Zernike moment were used in [20] for fingerprint recognition. In [21], a new orientation extraction baseline algorithm was proposed and the local and global features of fingerprints were analyzed. Based on the minutiae and pore extraction, a reference resolution was recommended for the AFIS in [22].

In this paper, we propose a new algorithm for fingerprint identification and verification based on the measurements from the statistical random variables using the extracting minutiae from the input image to locate the accurate core point as a reference point for the image. After calculating the Euclidean distance from the virtual core point for each extracted minutiae (bifurcation, termination), the statistical parameters like the mean, variance, skewness, and kurtosis are used for the detection of a fingerprint whether it comes from the same person or from a different one. Finally, seven invariant moment features are then used by providing the ultimate accuracy information to ensure the matching.

The paper is organized as follows: Section 2 deals with the preprocessing technique of an image. The mathematical model of the proposed algorithm in the detection of the virtual core point, the minutiae extraction technique, the moment invariant parameters, and the relation between statistical parameters and the recognition and verification system are also described in this section. Section 3 provides the results obtained from the simulation and performance evaluation in context of Section 2 and finally, Section 4 concludes the entire analysis.

\section{SYSTEM MODEL}

\subsection{Preprocessing}

The system model starts with a normal preprocessing approach. The enhancement process prepares to level up the image and makes the ridge pattern clarity better so that it can run at the maximum degree of favorable termination in further processing. We used a 500 dpi resolution of 
a fingerprint image that was $320 \times 480$ pixels in size (FBI standard [19]). In this paper, preprocessing is performed by gray-level normalization enhancement to compute the mean and variance in the pixel-wise manner as briefly described in [23].

The normalized image can be defined as:

$$
N(x, y)=\left\{\begin{array}{l}
m_{0}+\left\{\frac{v_{0}\left(\psi(x, y)-m_{i}\right)^{2}}{v_{i}}\right\}^{1 / 2} ; \text { if } \psi(x, y)>m \\
m_{0}-\left\{\frac{v_{0}\left(\psi(x, y)-m_{i}\right)^{2}}{v_{i}}\right\}^{1 / 2} ; \text { Otherwise }
\end{array}\right.
$$

where, $N(x, y)$ is the normalized gray-level value at pixel $(x, y), \psi_{i}(x, y)$ is the gray-level value at pixel $(x, y)$ of image $\psi_{i}$, and $m_{i}$ and $v_{i}$ represent the estimated mean and variance, respectively. $m_{0}$ and $v_{0}$ denote the desired mean and variance, respectively.

Therefore, for the $m \times n$ matrix of pixels, the mean and variance are represented respectively by:

$$
m(\psi)=\frac{1}{m n} \sum_{x=0}^{m-1} \sum_{y=0}^{n-1} \psi(x, y),
$$

and:

$$
v(\psi)=\frac{1}{m n} \sum_{x=0}^{m-1} \sum_{y=0}^{n-1}\{\psi(x, y)-m(\psi)\}^{2} .
$$

\subsection{Virtual Core Point Detection}

To speed up the overall process, the determination of the reference point is essential. After locating the point at its reference, it is observed that the core points are formed by a distinct pattern from the directional field. These curvatures of the ridges are similar to an elliptic loop [19]. In this paper, we propose a new system of reference point locating called the "virtual core point," by which we estimate the predefined area (ROI), where the actual core point or high curvatures must reside.

Let $\theta$ be the orientation parameter that can be computed by the local ridge orientation [6]. Here $\theta(x, y)$ represents the local ridge at pixel $(x, y)$ and is calculated in a non-overlapping block-wise manner. However, the orientation field, as based on the least mean square (LMS) algorithm is briefly described below in relation to the research outlined in this paper $[8,24]$ :

1) Separate and process the input image into non-overlapping blocks of size $w \times w$.

2) Find out the horizontal and vertical gradients $\varphi_{x}(x, y)$ and $\varphi_{y}(x, y)$ at each pixel $(x, y)$ by using the gradient operator.

3) Estimate the ridge orientation with the following equations: 


$$
V_{x}(x, y)=\sum_{u=x-\frac{w}{2}}^{x+\frac{w}{2}} \sum_{v=y-\frac{w}{2}}^{y+\frac{w}{2}} 2 \varphi_{x}(u, v) \varphi_{y}(u, v),
$$

and:

$$
V_{y}(x, y)=\sum_{u=x-\frac{w}{2}}^{x+\frac{w}{2}} \sum_{v=y-\frac{w}{2}}^{y+\frac{w}{2}}\left\{\varphi_{x}^{2}(u, v)-\varphi_{y}^{2}(u, v)\right\} .
$$

Consequently:

$$
\theta(x, y)=\frac{1}{2} \tan ^{-1}\left(\frac{V_{x}(x, y)}{V_{y}(x, y)}\right)
$$

However, we know that the slope of a curve on the point of the maxima is zero, but the slope to the immediate left of the maxima is positive and on the immediate right it is negative. The situation is reverse for the case of the minima, (i.e., the slope to the immediate left of the maxima is negative and on immediate right it is positive). The gradient of the immediate left and right points of the maxima are: $m_{1}=\left.(d y / d x)\right|_{\left(x_{1}, y_{1}\right)}$ and $m_{2}=\left.(d y / d x)\right|_{\left(x_{2}, y_{2}\right)}$ respectively. The change in gradient $\Delta m=\left|m_{1}-m_{2}\right|$ is the maximum for the case of the maxima or minima points of a ridge like Fig. 1, since the polarity of the slope is different on left and right side of the maxima or minima point.

However, by storing the ridge orientation mapping into an array and determining the maximum value of each row and their corresponding co-ordinate values, we can easily calculate the virtual core point.

\section{The algorithm for virtual core point detection is summarized as follows:}

1. Read the image.

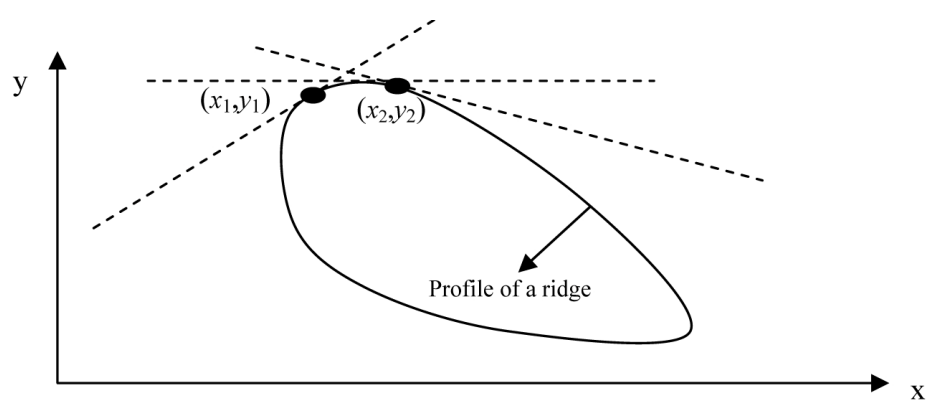

Fig. 1. Gradient of a ridge profile on the immediate left and right of the maxima 
2. Resize the image for uniform analysis.

3. Determine the ridge orientation curves of the image.

4. Store the ridge orientation image in an array $A(i, j) ; i=1,2,3, \ldots$ size of the $\operatorname{row}(M)$ and $j$ $=1,2,3, \ldots$ size of the column $(N)$ of the image.

5. Select the $i$-th row of the image $A(i,:)$ and determine $R(i, q)=a b s(A(i, q)-\mathrm{A}(i, q+1))$; where $q=1,2,3, \ldots N-1$.

6. Determine the maximum value of $R(i, q), z(i)=\max (R(i,:))$.

7. Determine the co-ordinate $\left(x_{\text {max }_{-},}, y_{\max _{-} i}\right)$ of the point of $z(i)$.

8. Repeat steps 5 to 7 for all rows to get $\left(x_{\text {max }_{-},}, y_{\max _{-}}\right) ; i=1,2,3, \ldots \mathrm{M}$.

9. Determine the following virtual core point:

$$
(\bar{X}, \bar{Y})=\left(\frac{\sum_{i=1}^{M} X_{\max _{-} i}}{M}, \frac{\sum_{i=1}^{M} Y_{\max _{-} i}}{M}\right) .
$$

\subsection{Minutiae Extraction}

To measure the minutiae information, the feature-analysis procedure is performed by considering the following two factors: 1) intensity distribution and 2) the relative arrangement of pixels in an image [17]. Here, the crossing number technique is applied to detect the bifurcation and termination points. This method identifies those ridge-maps by examining the local neighborhood of a $3 \times 3$ pixel window and calculates the number of pixels that intersect at the center of the pixel. The calculation is represented by the following equation $[16,25]$ :

$$
C(p i)=\sum_{k=1}^{8} p(k),
$$

where, $p(k)=0,1,2, \ldots, 7$ are the eight neighbor pixels of $C(p i)$ of a block taken as the ordered sequences. Therefore, the value of $C(p i)$ can possibly configure different cases of the ridge-maps shown in Fig. 2. If $C(p i)=5$ indicates that there exists a ridge bifurcation (Fig.2 (a)), $C(p i)=6$

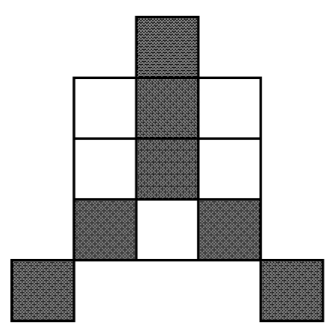

a) $C(p \mathrm{i})=5$

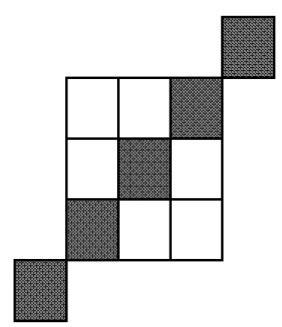

b) $C(p i)=6$

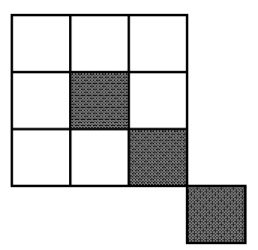

c) $C(p i)=7$

Fig. 2. Different pixel arrangements of a $3 \times 3$ pixel window a) ridge bifurcation $b$ ) an intermediate ridge pixel c) termination ridge 
indicates the intermediate ridge map (Fig.2(b)), and $C(p i)=7$ means that a ridge ending exists (Fig. 2(c)).

\subsection{Fingerprint Recognition}

This section deals with the recognition and verification performance to get an accurate matching output. In previous sections, we have already identified the virtual core point that almost indicates the centroid of the image and also extracts the bifurcation and termination points as minutiae. We are proposing a new approach to compare for recognition of fingerprint. The comparison is made with the following four important statistical parameters: mean, variance, kurtosis, and skewness. To determine the statistical distribution of minutiae points, we calculate the mean and variance of the distance between the minutiae points and the virtual core points like in Fig. 3.

Now, the mean and variance (the distance between the virtual core point and the minutiae point) is:

$$
\overline{l_{i}}=\sum_{i=1}^{N} \frac{l_{i}}{N},
$$

where:

$$
l_{i}=\sqrt{\left(v_{c x}-x_{i}\right)^{2}+\left(v_{c y}-y_{i}\right)^{2}}
$$

and:

$$
\operatorname{Var}(l)=\frac{\sum_{i=1}^{N}\left(l_{i}-\bar{l}_{i}\right)^{2}}{N} .
$$

Here $\left(v_{c x}, v_{c y}\right)$ are the coordinates of the virtual core point and $\left(x_{i}, y_{i}\right)$ are the coordinates of the

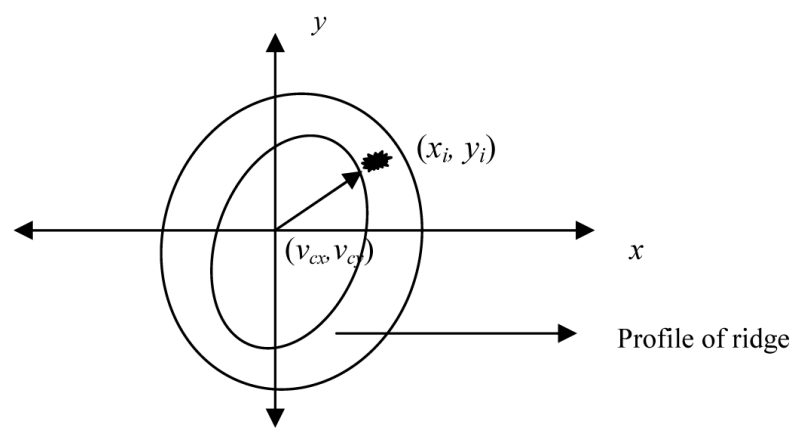

Fig. 3. The Euclidean distance measurement between the virtual core point and the extracted minutiae position 
minutiae. The skewness is a measure of the asymmetry of the probability distribution of a realvalued random variable $l_{i}$, which is expressed as:

$$
\alpha=\mathrm{E}\left[\left(\frac{l_{i}-\mu}{\sigma}\right)^{3}\right]=\frac{\mu_{3}}{\sigma^{3}}=\frac{\mathrm{E}\left(l_{i}^{3}\right)-3 \mu \sigma^{2}-\mu^{3}}{\sigma^{3}} .
$$

The kurtosis is a measure of the "peakedness" (the ratio of the variance and mean) of the probability distribution of a real-valued random variable, which is expressed as:

$$
\beta=\frac{\mu_{4}}{\sigma^{4}}-3
$$

where $\mu$ is the mean and $\sigma$ is the standard deviation of the random variable $l_{i}$.

\section{E. Fingerprint Verification}

Computing the invariant moments that are insensitive to deformation, especially for rotation, now validates the efficiency of the fingerprint recognition. Therefore, if $f(x, y)$ is a digital image, the moment of order $(p+q)$ is defined as:

$$
m_{p q}=\int_{-\infty}^{+\infty} \int_{-\infty}^{+\infty} x^{p} y^{q} f(x, y) d x d y \quad ; \mathrm{p}, \mathrm{q}=0,1,2 \ldots
$$

A set of seven invariant moments can be applied for invariant pattern identification as below:

$$
\begin{aligned}
& \delta_{1}=\eta_{20}+\eta_{02}, \\
& \delta_{2}=\left(\eta_{20}-\eta_{02}\right)^{2}+4 \eta_{11}^{2}, \\
& \delta_{3}=\left(\eta_{30}-3 \eta_{12}\right)^{2}+\left(3 \eta_{21}-\eta_{03}\right)^{2}, \\
& \delta_{4}=\left(\eta_{30}+\eta_{12}\right)^{2}+\left(\eta_{21}+\eta_{03}\right)^{2}, \\
& \delta_{5}=\left(\eta_{30}-3 \eta_{12}\right)\left(\eta_{30}+\eta_{12}\right)\left[\left(\eta_{30}+\eta_{12}\right)^{2}-3\left(\eta_{21}+\eta_{03}\right)^{2}\right]+ \\
& \left(3 \eta_{21}-\eta_{03}\right)\left(\eta_{21}+\eta_{03}\right)\left[3\left(\eta_{30}+\eta_{12}\right)^{2}-\left(\eta_{21}+\eta_{03}\right)^{2}\right] \\
& \delta_{6}=\left(\eta_{20}-\eta_{02}\right)\left[\left(\eta_{30}+\eta_{12}\right)^{2}-\left(\eta_{21}+\eta_{03}\right)^{2}\right]+4 \eta_{11}\left(\eta_{30}+\eta_{12}\right)\left(\eta_{21}+\eta_{03}\right), \\
& \delta_{7}=\left(3 \eta_{21}-\eta_{03}\right)\left(\eta_{30}+\eta_{12}\right)\left[\left(\eta_{30}+\eta_{12}\right)^{2}-3\left(\eta_{21}+\eta_{03}\right)^{2}\right]+ \\
& \left(3 \eta_{12}-\eta_{30}\right)\left(\eta_{21}+\eta_{03}\right)\left[3\left(\eta_{30}+\eta_{12}\right)^{2}-\left(\eta_{21}+\eta_{03}\right)^{2}\right] .
\end{aligned}
$$

\section{Experiments and Performance Evaluation}

In this section, more than 200 fingerprints were taken from different people for analysis and verification. In Fig. 4(a), the fingerprint of Person-1 with a single core at the middle was taken. Their ridge orientations are shown with dotted lines. Fig. 4(b) shows the original image, its ridge 


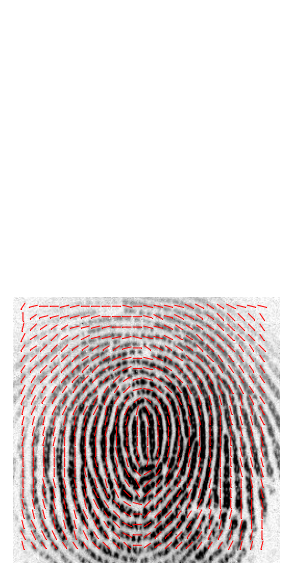

(a)
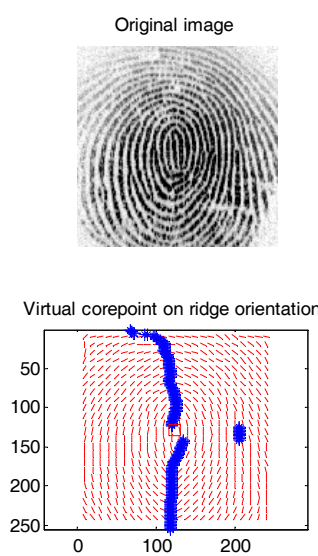

(b)

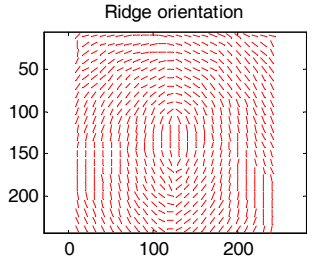

Virtual corepoint on original image

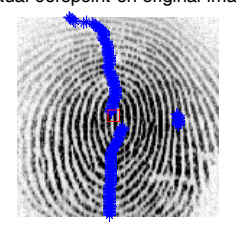

Fig. 4. (a) Fingerprint of Person-1 with a single core at the middle, (b) Virtual core point of Person-1

orientation, virtual core point on its ridge orientation, and virtual core point on its original image. The profile of $\left(x_{\max }, y_{\max }\right) ; i=1,2,3, . ., M$; follows the maxima and minima points of the ridge orientation as shown by dark lines. The virtual core point $(\bar{X}, \bar{Y})$ that was detected by the proposed algorithm is indicated by the square, which is exactly on the core point.

A similar analysis was done for three other types of images. For the case of a fingerprint with two core points, the virtual core point was found to be approximately at the middle point of the connecting line of two original core points, as shown in Fig. 5(b). When the core point was located in one corner, the virtual core point shifted diagonally a little along the opposite corner, as visualized in Fig. 6(b). Finally, when the fingerprint did not have a core point, then $(\bar{X}, \bar{Y})$ indicates the point vicinity of the centroid of the image, as shown in Fig. 7(b).

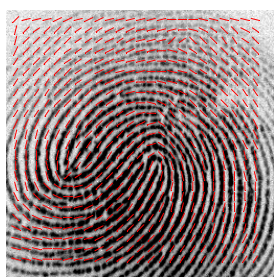

(a)
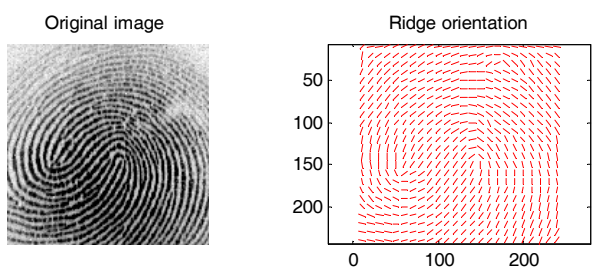

Virtual corepoint on original image
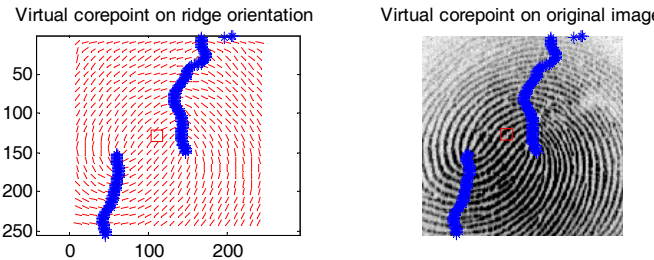

(b)

Fig. 5. (a) Fingerprint of Person-2 with two cores, (b) Virtual core point of Person-2 


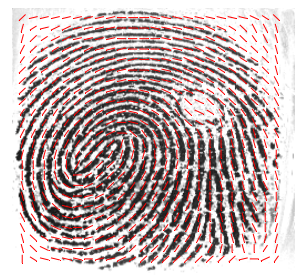

(a)

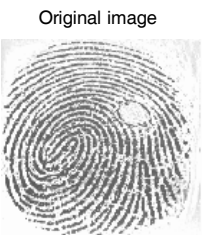

Virtual corepoint on ridge orientation

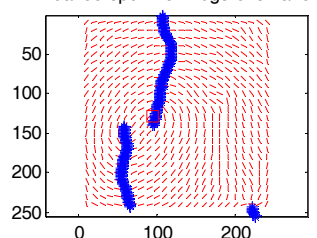

Fig. 6. (a) Fingerprint of Person-3 with a single core at one corner, (b) Virtual core point of Person-3

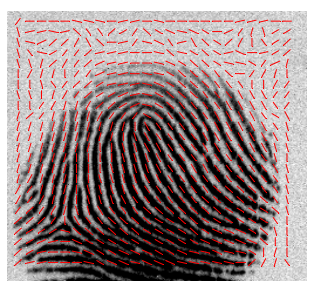

(a)
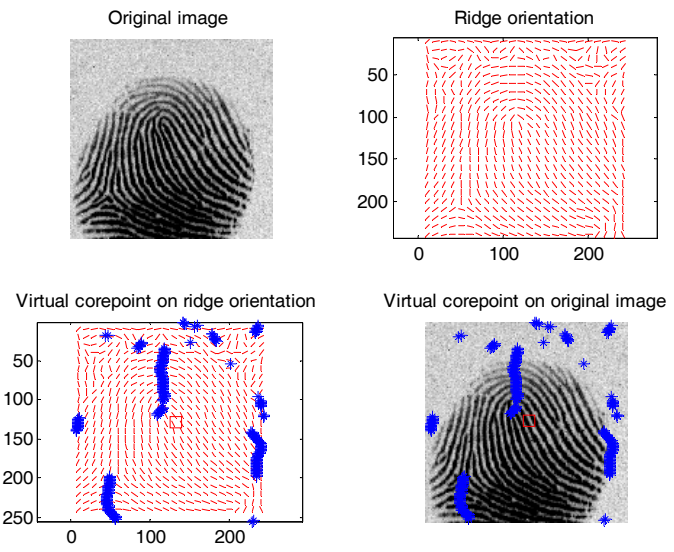

(b)
Virtual corepoint on original image

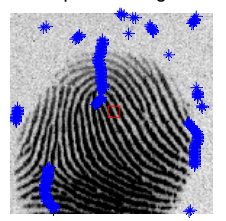

(b)

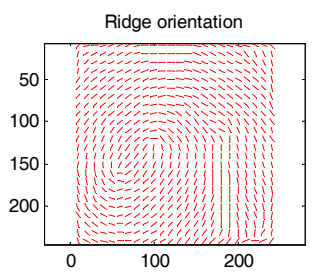

Virtual corepoint on original image

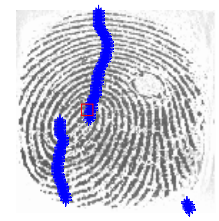

)

Fig. 7. (a) Fingerprint of Person-4 without a core point, (b) Virtual core point of Person-4

Now, for the extract statistical parameters and moment invariants, we selected 8 fingerprints from the same person, as shown in Table-1(a) and Table-1(b). Figure 8 shows the original image, the enhanced version of the image, the image after thinning, bifurcation points on the image, bifurcation points on the Cartesian co-ordinate system, and the invariant moments of the same person case. The variation of the parameters is visualized in Figs. 8(a) and 8(b), which arises because of different orientations of the image. A similar analysis is shown in Figs. 9(a) and 9(b) for the case of termination points. Finally, Figs. 10 and 11 show the analysis of different individuals for the cases of the bifurcation and termination points respectively.

To calculate the statistical parameters and moment invariants based on bifurcation points, we selected 8 fingerprints from the same person, which is shown in Table-1(a) and Table-1(b) below. 


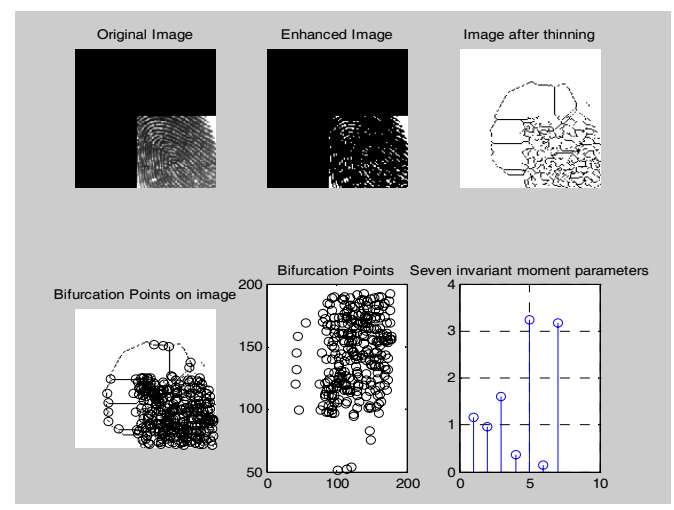

(a) Fingerprint-1

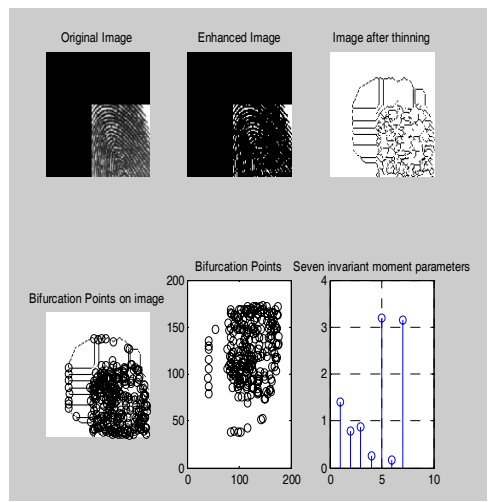

(b) Fingerprint-2

Fig. 8. Bifurcation points and invariant moment parameters of fingerprint of the same person

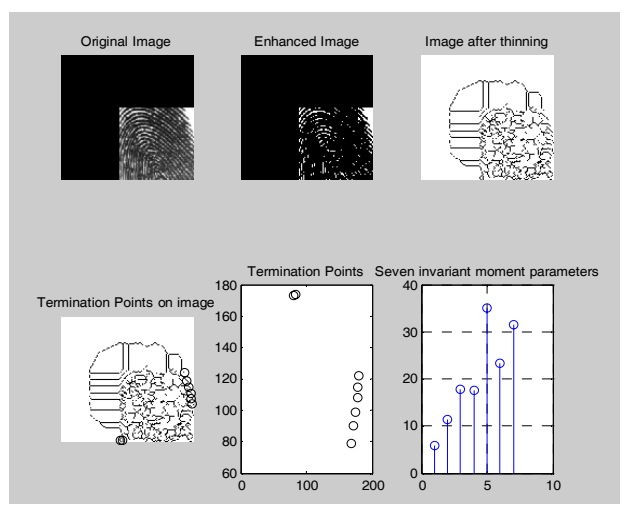

(a) Fingerprint-1

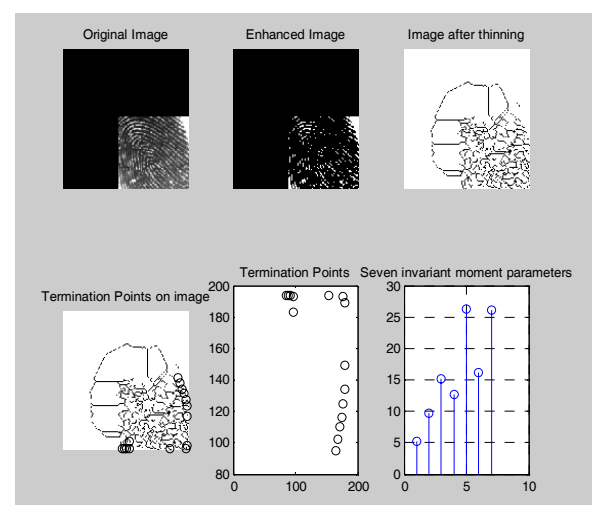

(b) Fingerprint-2

Fig. 9. Termination points and invariant moment parameters of the fingerprint of the same person

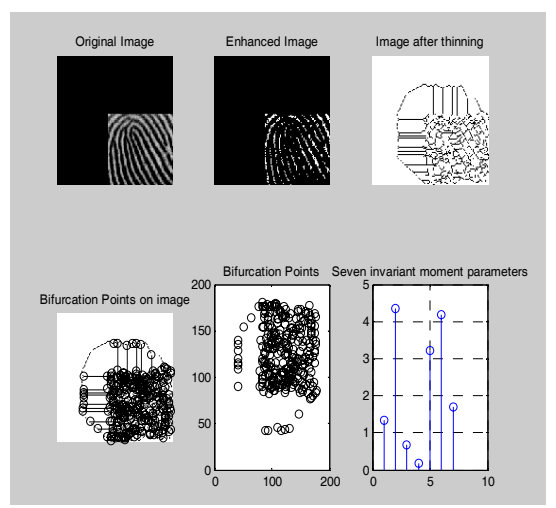

(a) Person-1

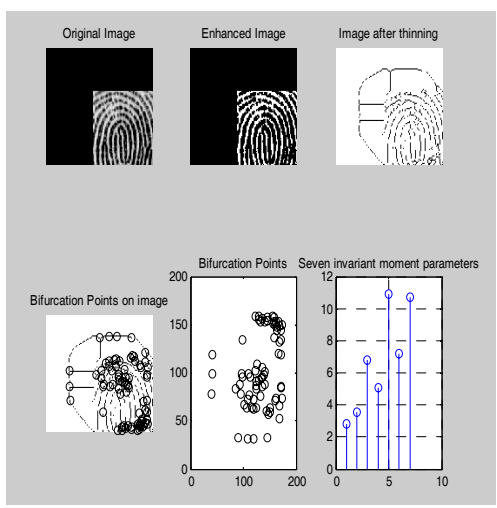

(b) Person-2

Fig. 10. The bifurcation points and invariant moment parameters of the fingerprints of different persons 


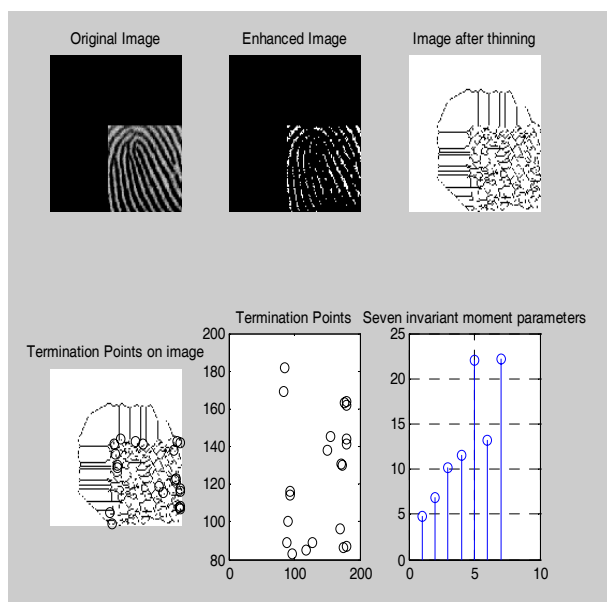

(a) Person-1

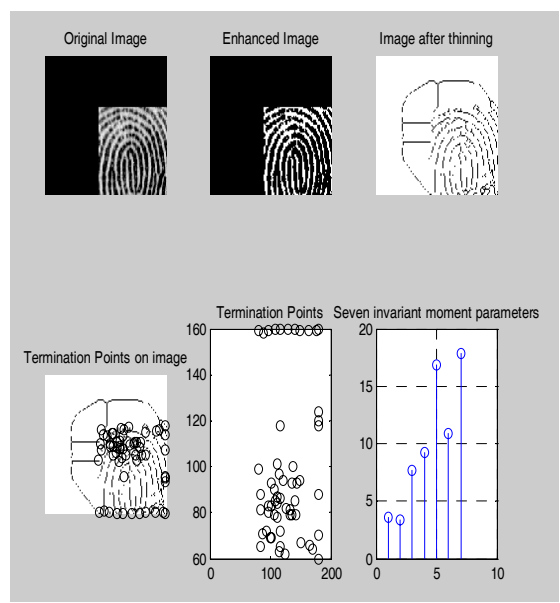

(b) Person-2

Fig. 11. Termination points and invariant moment parameters of the fingerprints of different persons

The maximum deviation of the mean distance $\overline{l_{i}}$ is found to be $9.0205 \%$ and the minimum deviation is only $3.1662 \%$. The percentage deviation of the variance is found as $\geq 34.8841 \%$. The kurtosis and skewness are found to be very close to each other (Table-1(a)). Finally, we observe from Table-1(b) that the invariant moments are also found to be very close to each other for all 8 images of the same person case.

Tables-1(c) and 1(d) show a similar analysis for the case of fingerprints from 6 different people. Here, the maximum deviation of the mean distance is $60.9210 \%$ and its minimum value is $16.4016 \%$. The percentage deviation of the variance is found to be $\geq 84.2020 \%$. Other statistical parameters, kurtosis, and skewness are also fluctuated for the case of different individuals, as is visualized in Table-1(c). Table-1(d) gives the seven invariant parameters that are quite far apart from one another. The analysis described above is done for a bifurcation points' case. Let us now concentrate on the case of termination points.

A high percentage of acceptance is obtained by extracting the termination points from the 8 images of the same person, as shown in Table-2(a). The maximum deviation of mean distance $\overline{l_{i}}$ is found to be $8.8232 \%$ and the minimum derivation is only $1.4296 \%$, where the deviation is

Table 1. (a) Fingerprints from the Same Person: Bifurcation Point

\begin{tabular}{c|c|c|c|c|c|c|c}
\hline $\begin{array}{c}\text { Same Person } \\
\text { Fingerprint \# }\end{array}$ & $\left(v_{\mathrm{cx}}, v_{\mathrm{cy}}\right)$ & Mean $\left(\overline{l_{i}}\right)$ & $\begin{array}{c}\text { Deviation } \\
\%(\mathrm{mean})\end{array}$ & $\begin{array}{c}\text { Variance } \\
\operatorname{Var}(l)\end{array}$ & $\begin{array}{c}\text { Deviation\% } \\
(\mathrm{var})\end{array}$ & Kurtosis & Skewness \\
\hline 1 & $(121.5313,128.5000)$ & 40.9063 & 6.1343 & 290.6462 & 34.8841 & 3.9926 & 0.6013 \\
\hline 2 & $(123.7305,128.5000)$ & 40.8115 & 6.3518 & 328.6721 & 26.3649 & 4.2202 & 0.7298 \\
\hline 3 & $(144.1289,128.5000)$ & 43.5796 & 0 & 382.4363 & 14.3196 & 3.3050 & 0.4699 \\
\hline 4 & $(146.9785,128.5000)$ & 41.4034 & 4.9936 & 446.3523 & 0 & 3.0474 & 0.5186 \\
\hline 5 & $(120.8926,128.5000)$ & 42.1998 & 3.1662 & 347.6230 & 22.1191 & 3.2821 & 0.4842 \\
\hline 6 & $(113.5098,128.5000)$ & 41.4644 & 4.8536 & 424.3420 & 4.9311 & 2.5391 & 0.3855 \\
\hline 7 & $(129.6680,128.5000)$ & 39.6485 & 9.0205 & 315.1974 & 29.3837 & 4.1310 & 0.6740 \\
\hline 8 & $(133.1387,128.5000)$ & 40.5043 & 7.0567 & 317.0364 & 28.9717 & 4.1574 & 0.7642 \\
\hline
\end{tabular}


Table 1. (b) Moment Invariant: Bifurcation Point [Same Person]

\begin{tabular}{c|c|c|c|c|c|c|c}
\hline $\begin{array}{c}\text { Same Person } \\
\text { Fingerprint } \#\end{array}$ & $\delta_{1}$ & $\delta_{2}$ & $\delta_{3}$ & $\delta_{4}$ & $\delta_{5}$ & $\delta_{6}$ & $\delta_{7}$ \\
\hline 1 & 1.4326 & 1.3892 & 1.0091 & 0.0927 & 3.2068 & 0.8305 & 5.6830 \\
\hline 2 & 1.4050 & 0.7935 & 0.8687 & 0.2512 & 3.1985 & 0.1602 & 3.1537 \\
\hline 3 & 1.1695 & 0.9642 & 1.5925 & 0.3609 & 3.2348 & 0.1522 & 3.1773 \\
\hline 4 & 1.0227 & 2.7453 & 1.1449 & 0.3307 & 2.0134 & 3.6532 & 1.1504 \\
\hline 5 & 1.3195 & 0.9366 & 0.8912 & 0.5666 & 3.1466 & 0.0921 & 0.0997 \\
\hline 6 & 1.1071 & 1.2843 & 1.3228 & 1.0484 & 2.7955 & 1.6968 & 3.9722 \\
\hline 7 & 1.1816 & 3.6928 & 0.6940 & 0.2150 & 0.7000 & 2.5089 & 3.7690 \\
\hline 8 & 1.2083 & 0.8091 & 0.9509 & 0.1709 & 1.7652 & 0.7464 & 3.1486 \\
\hline
\end{tabular}

Table 1. (c) Fingerprints from Different Persons: Bifurcation Point

\begin{tabular}{c|c|c|c|c|c|c|c}
\hline Person & $\left(v_{c x}, v_{c y}\right)$ & Mean $\left(\overline{l_{i}}\right)$ & $\begin{array}{c}\text { Deviation } \\
\%(\text { mean })\end{array}$ & Variance Var $(l)$ & $\begin{array}{c}\text { Deviation\% } \\
(\text { var })\end{array}$ & Kurtosis & Skewness \\
\hline 1 & $(103.5352,128.5000)$ & 52.7955 & 39.5732 & 529.6936 & 69.6277 & 2.4390 & 0.0795 \\
\hline 2 & $(156.0156,128.5000)$ & 87.3710 & 0 & $1.7440 \mathrm{e}+003$ & 0 & 1.8315 & -0.6773 \\
\hline 3 & $(128.5566,128.5000)$ & 73.0408 & 16.4016 & 445.2161 & 74.4716 & 1.4689 & -0.5507 \\
\hline 4 & $(123.1230,128.5000)$ & 34.1437 & 60.9210 & 275.5168 & 84.2020 & 4.8147 & 0.9546 \\
\hline 5 & $(143.3262,128.5000)$ & 42.6792 & 51.1518 & 417.9483 & 76.0351 & 4.0895 & 0.7130 \\
\hline 6 & $(139.2988,128.5000)$ & 37.6123 & 56.9510 & 383.1620 & 78.0297 & 3.7845 & 0.8488 \\
\hline
\end{tabular}

Table 1. (d) Moment Invariant: Bifurcation Point [Different Persons]

\begin{tabular}{c|c|c|c|c|c|c|c}
\hline Person & $\delta_{1}$ & $\delta_{2}$ & $\delta_{3}$ & $\delta_{4}$ & $\delta_{5}$ & $\delta_{6}$ & $\delta_{7}$ \\
\hline 1 & 1.3570 & 2.8472 & 0.5257 & 1.9656 & 2.5264 & 0.5352 & 3.0647 \\
\hline 2 & 5.8812 & 9.5729 & 15.9390 & 15.1832 & 30.7116 & 19.9490 & 30.1766 \\
\hline 3 & 3.7444 & 6.8444 & 9.6515 & 8.2579 & 16.9799 & 10.7423 & 16.7183 \\
\hline 4 & 0.8447 & 1.9463 & 1.0449 & 0.1882 & 3.2791 & 3.3876 & 0.3819 \\
\hline 5 & 1.8785 & 0.6710 & 4.4847 & 3.7874 & 6.8223 & 4.0286 & 7.8649 \\
\hline 6 & 0.7734 & 2.9463 & 0.3443 & 0.2457 & 3.1929 & 3.7931 & 1.9674 \\
\hline
\end{tabular}

Table 2. (a) Fingerprints from the Same Person: Termination Point

\begin{tabular}{c|c|c|c|c|c|c|c}
\hline $\begin{array}{c}\text { Same Person } \\
\text { Fingerprint }\end{array}$ & $\left(v_{c x}, v_{c y}\right)$ & Mean $\left(\overline{l_{i}}\right)$ & $\begin{array}{c}\text { Deviation } \\
\%(\text { mean })\end{array}$ & Variance Var $(l)$ & $\begin{array}{c}\text { Deviation } \\
\%(\text { var })\end{array}$ & Kurtosis & Skewness \\
\hline 1 & $(121.5313,128.5000)$ & 59.6444 & 0 & 42.7336 & 91.3727 & 2.4639 & 0.4248 \\
\hline 2 & $(128.6875,128.5000)$ & 55.3416 & 7.2141 & 145.1433 & 70.6977 & 4.7421 & 0.6011 \\
\hline 3 & $(144.1289,128.5000)$ & 57.9501 & 2.8407 & 495.3312 & 0 & 1.2484 & 0.0317 \\
\hline 4 & $(145.1621,128.5000)$ & 57.0035 & 4.4277 & 236.4450 & 52.2653 & 1.6773 & 0.0991 \\
\hline 5 & $(120.8926,128.5000)$ & 58.7917 & 1.4296 & 115.2876 & 76.7251 & 2.3152 & -0.7138 \\
\hline 6 & $(112.4902,128.5000)$ & 54.5877 & 8.4781 & 365.1264 & 26.2864 & 2.0372 & -0.0187 \\
\hline 7 & $(129.6680,128.5000)$ & 57.5407 & 3.5271 & 63.0955 & 87.2620 & 2.9603 & -0.5710 \\
\hline 8 & $(108.1719,128.5000)$ & 54.3699 & 8.8432 & 408.5902 & 17.5117 & 1.5986 & -0.0640 \\
\hline
\end{tabular}


Table 2. (b) Moment Invariant: Termination Point [Same Person]

\begin{tabular}{c|c|c|c|c|c|c|c}
\hline $\begin{array}{c}\text { Same Person } \\
\text { Fingerprint \# }\end{array}$ & $\delta_{1}$ & $\delta_{2}$ & $\delta_{3}$ & $\delta_{4}$ & $\delta_{5}$ & $\delta_{6}$ & $\delta_{7}$ \\
\hline 1 & 5.4968 & 8.3195 & 9.9524 & 14.2666 & 26.5579 & 18.3824 & 24.2418 \\
\hline 2 & 4.7010 & 8.6017 & 13.9424 & 13.5922 & 27.3593 & 17.8391 & 23.4840 \\
\hline 3 & 5.2875 & 9.6145 & 15.1097 & 12.6305 & 26.2356 & 16.0970 & 26.0565 \\
\hline 4 & 4.4214 & 7.7427 & 12.9713 & 10.9839 & 23.1350 & 15.1829 & 21.9157 \\
\hline 5 & 5.3105 & 9.6977 & 15.6528 & 14.5803 & 29.6965 & 19.3197 & 26.3210 \\
\hline 6 & 5.1543 & 5.7286 & 13.6228 & 13.1461 & 23.5320 & 15.2334 & 26.7147 \\
\hline 7 & 4.6105 & 7.6348 & 12.2008 & 11.8036 & 23.9052 & 15.0002 & 23.2002 \\
\hline 8 & 5.5351 & 9.3832 & 16.5665 & 14.3271 & 26.6182 & 16.6053 & 29.9383 \\
\hline
\end{tabular}

Table 2. (c) Fingerprints from Different Persons: Termination Point

\begin{tabular}{c|c|c|c|c|c|c|c}
\hline Person & $\left(v_{c x}, v_{c y}\right)$ & Mean $\left(\overline{l_{i}}\right)$ & $\begin{array}{c}\text { Deviation\% } \\
(\text { mean })\end{array}$ & $\begin{array}{c}\text { Variance } \\
\operatorname{Var}(l)\end{array}$ & $\begin{array}{c}\text { Deviation } \\
\%(\text { var })\end{array}$ & Kurtosis & Skewness \\
\hline 1 & $(103.5352,128.5000)$ & 57.5553 & 11.6766 & 575.2582 & 0 & 2.2012 & -0.4043 \\
\hline 2 & $(156.0156,128.5000)$ & 43.5372 & 33.1886 & 450.6680 & 21.6581 & 2.6706 & 0.2996 \\
\hline 3 & $(128.5566,128.5000)$ & 30.4498 & 53.2723 & 195.5328 & 66.0096 & 3.6021 & 0.4665 \\
\hline 4 & $(123.1230,128.5000)$ & 32.2077 & 50.5746 & 104.1123 & 81.9016 & 1.9627 & 0.0918 \\
\hline 5 & $(143.3262,128.5000)$ & 65.1643 & 0 & 265.6094 & 53.8278 & 7.2917 & -0.5438 \\
\hline 6 & $(139.2988,128.5000)$ & 37.5642 & 42.3546 & 466.6735 & 18.8758 & 1.7793 & -0.3681 \\
\hline
\end{tabular}

Table 2. (d) Moment Invariant: Termination Point [Different Persons]

\begin{tabular}{c|c|c|c|c|c|c|c}
\hline Person & $\delta_{1}$ & $\delta_{2}$ & $\delta_{3}$ & $\delta_{4}$ & $\delta_{5}$ & $\delta_{6}$ & $\delta_{7}$ \\
\hline 1 & 3.2406 & 0.4586 & 5.9501 & 5.9032 & 11.7897 & 5.4670 & 11.0068 \\
\hline 2 & 1.9857 & 0.6510 & 2.9685 & 0.4851 & 2.1926 & 0.4866 & 3.1937 \\
\hline 3 & 1.6663 & 1.2951 & 3.4495 & 2.1097 & 5.0411 & 0.0328 & 4.8077 \\
\hline 4 & 2.1279 & 3.3155 & 5.5606 & 5.7737 & 11.4098 & 7.3289 & 10.5148 \\
\hline 5 & 4.2200 & 6.8993 & 10.9054 & 10.6013 & 20.7172 & 14.3673 & 21.4906 \\
\hline 6 & 3.0612 & 0.2948 & 8.4007 & 7.4194 & 12.7579 & 7.8769 & 15.3266 \\
\hline
\end{tabular}

apparently small. The percentage deviation of the variance is found to be $\geq 91.3727 \%$. The kurtosis and skewness are found very close to each other. Finally, the invariant moments also assure close resemblance data for each order of all 8 images from the same person, as observed in Table-2(b).

Similarly, a better comparison assurance can be seen in Table-2(c) and Table-2(d) for the case of different individuals. The value of the mean, variance, kurtosis, and skewness vary irregularly for the 6 different fingerprints from 6 people. For example, the maximum and minimum deviations of the mean distances are $53.2723 \%$ and $11.6766 \%$, respectively. In addition, the invariant moment parameters in the order of 1 to 7 make a transparent difference for different persons.

Similarly, a better comparison assurance can be seen in Table-2(c) and Table-2(d) for the case of different individuals. The value of the mean, variance, kurtosis, and skewness vary irregularly for the 6 different fingerprints from 6 persons. For example, the maximum and minimum deviations of the mean distances are $53.2723 \%$ and $11.6766 \%$, respectively. In addition, the invariant 
moment parameters in the order of 1 to 7 make a transparent difference for different persons.

\section{CONCLUSION}

We have proposed an enhanced minutiae-based algorithm for the fingerprint identification system using statistical and moment-based parameters. The root of the analysis is based on the newly proposed virtual core-point of a fingerprint and on the region of interest (ROI), which is adjacent to the core point. The overall experimental results have demonstrated that our proposed method exhibits an encouraging improvement in the detection of fingerprints from an image pool. The work can be extended based on a two-dimensional spectrum of gray scale fingerprint on the ROI by using a continuous wavelet transform to get a better resolution.

\section{REFERENCES}

[1] S. C. Dass and A.K. Jain, "Fingerprint-Based Recognition," Technometrics, 2007, pp.262-276.

[2] M. K. Khan, "Fingerprint Biometric-based Self-Authentication and Deniable Authentication Schemes for the Electronics World," IETE Tech Rev, Vol.26, No.3, 2009, pp.191-195.

[3] S. W. Lee and B. H. Nam, "Fingerprint Recognition Using Wavelet Transform and Probabilistic Neural Network," International Joint Conference on Neural Network, Vol.5, 1999, pp.3276-3279.

[4] A. K. Jain, S. Prabhakar and L. Hong, "A Multichannel Approach to Fingerprint Classification," IEEE Transactions on PAMI, Vol.21, No.4, Apr., 1999, pp.348-359.

[5] J. C. Yang, D. S. Park, “A Fingerprint Verification Algorithm Using Tessellated Invariant Moment Features,” Neurocomputing, 71, 2008, pp.1939-1946.

[6] Q. Zhao, D. Zhang, L. Zhang, N. Luo, “Adaptive Fingerprint Pore Modeling and Extraction,” Pattern Recognition, 43, 2010, pp.2833-2844.

[7] D. Maltoni, D. Maio, A. K. Jain and S. Prabhakar, "Handbook of Fingerprint Recognition," Springer, Berlin, 2003, pp.135-137.

[8] A.K. Jain, S. Prabhakar, L. Hong and S. Pankanti, "Filterbank-based Fingerprint Matching," IEEE Transactions on Image Processing, Vol.9, No.5, 2000, pp.846-859.

[9] M. S. Khalil, D. Muhammad, M. K. Khan, K. Alghathbar, "Singular Points Detection Using Fingerprint Orientation Field Reliability," International Journal of Physical Sciences, Vol.5, No.4,2010, pp.352-357.

[10] C. L. Deepika, A. Kandaswamy, C. Vimal and B Sathish, "Invariant Feature Extraction from Fingerprint Biometric Using Pseudo Zernike Moments," International Journal of Computer Communication and Information System, Vol.2, No.1, 2010, pp.104-108.

[11] K. A. Nagaty, "An Adaptive Hybrid Energy-based Fingerprint Matching Technique," Image and Vision Computing, 23, 2005, pp.491-500.

[12] L. Nanni, A Lumini, “A Hybrid Wavelet-based Fingerprint Matcher," Pattern Recognition, Vol.40, No.11,2007, pp.3146-3151.

[13] A. Ross, A. K. Jain, J. Reisman, “A Hybrid Fingerprint Matcher,” Pattern Recognition, Vol.36, No.7, 2003, pp.1661-1673.

[14] F. Benhammadi, M. N. Amirouche, H. Hentous, K. B. Beghdad and M. Aissani, "Fingerprint Matching From Minutiae Texture Maps,” Pattern Recognition, Vol.40, No.1, 2007, pp.189-97.

[15] M. Tico, P. Kuosmanen and J. Saarinen, "Wavelet Domain Features for Fingerprint Recognition," Electronics Letter, Vol.37, No.1, 2001, pp.21-22.

[16] J. L. Garcia, G. S. Perez, T. Medina, "Invariant Moments Applied to Fingerprint Recognition," International Journal of Circuits, Systems and Signal Processing, Vol.2, 2008, pp.180-187.

[17] M. S. Khalil, D. Muhammad, M. K. Khan, K. Alghathbar, "Fingerprint Verification Based on Statistical Analysis," International Conference on Future Information Technology, 2010, pp.1-5 
[18] W. Zhang and Y. Wang, "Singular Point Detection in Fingerprint Image," Asian Conference on Computer Vision, Vol.2, Jan 2002, pp.793-796.

[19] A. Mishra and M. Shandilya, "Fingerprint Core Point Detection Using Gradient Field Mask," International Journal of Computer Applications, Vol.2, No.8, 2010, pp.19-23.

[20] J. Yang, N. Xiong, A. V. Vasilakos, Z. Fang, D. Park, X. Xu, S. Yoon, S. Xie, and Y. Yang, "A Fingerprint Recognition Scheme Based on Assembling Invariant Moments for Cloud Computing Communications," IEEE Systems Journal, 2011.

[21] Turroni, D. Maltoni, R. Cappelli and D. Maio, "Improving Fingerprint Orientation Extraction," IEEE Transactions on Information Forensics and Security, Vol.60, No.3, 2011, pp.1002-1013.

[22] D. Zhang, F. Liu, Q. Zhao, G. Lu and N. Luo, "Selecting a Reference High Resolution For Fingerprint Using Minutiae and pores," IEEE Transactions on Instrumentation and Measurement, Vol.60, No.3, 2011, pp.863-871.

[23] A. Julasayvake and S. Choomchuay, "An Algorithm for Fingerprint Core Point Detection," International Symposium on Signal Processing and Its Applications, 2007, pp.1-4.

[24] A. Jain, L. Hong, and R. Bolle, "On-line Fingerprint Verification," IEEE Transactions on Pattern Analysis and Machine Intelligence, Vol.19, 1997, pp.302-314.

[25] J. Leon, G. Sanchez, G. Aguilar, L. Toscano, H. Perez and J. M. Ramirez, "Fingerprint Verification Applying Invariant Moments," IEEE International Midwest Symposium on Circuits and Systems, 2009, pp.751-757.

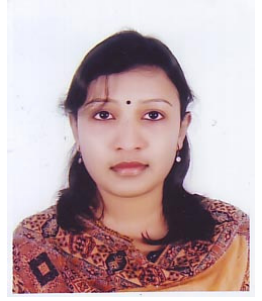

\section{Sarnali Basak}

Sarnali Basak received her B.Sc. (Hons.) in Computer Science and Engineering from Jahangirnagar University in Savar Dhaka, Bangladesh in 2008. She is studying for her MS in the Department of Computer Science and Engineering at Jahangirnagar University in Savar, Dhaka. She is working as a lecturer in the Department of Computer Science and Engineering at Prime University in Dhaka1216, Bangladesh. She received an NSICT fellowship from the Ministry of Science and Information \& Communication Technology, Bangladesh for her MS research in 2011. Her research fields are Image Processing, Pattern Recognition, Artificial Intelligence, Neural Networks and Biometrics.

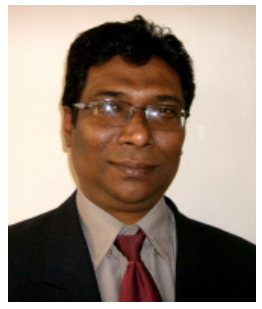

\section{Md. Imdadul Islam}

Md. Imdadul Islam received his B.Sc. and M.Sc Engineering in Electrical and Electronic Engineering from the Bangladesh University of Engineering and Technology in Dhaka, Bangladesh in 1993 and 1998 respectively and received his Ph.D from the Department of Computer Science and Engineering at Jahangirnagar University in Dhaka, Bangladesh in the field of Network Traffic Engineering, in 2010. He is now working as a Professor at the Department of Computer Science and Engineering, Jahangirnagar University in Savar, Dhaka, Bangladesh. Previously, he worked as an Assistant Engineer in Sheba Telecom (Pvt.) LTD (A joint venture company between Bangladesh and Malaysia, for Mobile Cellular and WLL), from Sept. 1994 to July 1996. Dr. Islam has a very good field experience in the installation of Radio Base Stations and Switching Centers for WLL. His research fields are network traffics, wireless communications, wavelet 
transforms, OFDMA, WCDMA, adaptive filter theory, ANFIS, and array antenna systems. He has more than a hundred research papers in national and international journals and conference proceedings.

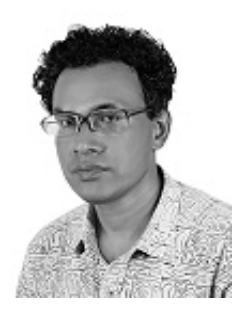

\section{R. Amin}

M. R. Amin received his B.S. and M.S. degrees in Physics from Jahangirnagar University in Dhaka, Bangladesh in 1984 and 1986, respectively and his Ph.D. degree in Plasma Physics from the University of St. Andrews in the U.K. in 1990. $\mathrm{He}$ is a Professor of Electronics and Communications Engineering at the East West University in Dhaka, Bangladesh. He served as a Post-Doctoral Research Associate in Electrical Engineering at the University of Alberta, Canada from 1991-1993. He was an Alexander von Humboldt Research Fellow at the MaxPlanck Institute for Extraterrestrial Physics at Garching in Munich, Germany during 1997-1999. Dr. Amin was awarded the Commonwealth Postdoctoral Fellowship in 1997. Besides these awards, he has also received several awards for his research, including the Bangladesh Academy of Science Young Scientist Award for the year 1996 and the University Grants Commission Young Scientist Award for 1996. His current research fields are wireless communications and networks and also nonlinear plasma dynamics. He is a member of the IEEE. 